\title{
Semiclassical transition probabilities by an asymptotic evaluation of the $S$ matrix for elastic and inelastic collisions. Bessel uniform approximation*
}

\author{
J. R. Stine and R. A. Marcus \\ Department of Chemistry, University of Illinois, Urbana, Illinois 61801
}

(Received 29 June 1973)

\begin{abstract}
It has been observed in the past that the usual Airy uniform approximation gives probabilities greater than one, especially for near elastic collisions. By mapping the phase onto $-\zeta \cos y+k y+A$ rather than $(1 / 3) y^{3}-\zeta y+A$ one obtains a uniform approximation involving Bessel functions of the first kind, which approaches unity for the elastic collision. This Bessel uniform approximation is no more complicated than the Airy and also gives good agreement with exact quantum results, even if probabilities are large.
\end{abstract}

\section{INTRODUCTION}

There has been considerable interest recently in the "exact" semiclassical treatment of inelastic collisions. ${ }^{1-5}$ An integral formulation has been given for the $S$ matrix ${ }^{12, b, 2 b}$ which is of the form

$$
S=\int_{0}^{1} g(x) e^{i f(\alpha, x)} d x .
$$

By approximating $f(\alpha, x)$ by a quadratic ("semiclassical result") or by a cubic ("Airy result") one obtains approximations for $S$ valid for two stationary phase points far apart or close together, respectively. By mapping $f(\alpha, x)$ onto a cubic ("Airy uniform") ${ }^{6}$ a uniform approximation is obtained which is valid regardless of whether the stationary phase points are far apart or close together, and indeed, it has produced excellent results.

In the limit of a near elastic collision, however, even the Airy uniform approximation breaks down and results in probabilities greater than one. This can be seen quite easily if one approximates ${ }^{20,32,7}$ $d f(\alpha, x) / d x$, for the near elastic collision, as one may do, by

$$
d f(\alpha, x) / d x=2 \pi \epsilon \sin 2 \pi x,
$$

where $\epsilon \rightarrow 0$ for an elastic collision, and if one assumes $g(x)$ to be unity for a near-elastic collision. The resulting expression for the probability becomes $^{8}$

$$
P=|S|^{2}=2(3 / 2)^{1 / 3} \epsilon^{-2 / 3} \mathrm{Ai}^{2}\left[-\left(\frac{3}{2} \epsilon\right)^{2 / 3}\right] .
$$

In the limit of $\epsilon$ approaching zero, the collision thereby becoming elastic, the probability becomes unbounded since $\mathrm{Ai}(0)$ is nonzero. This singularity in the Airy uniform expression has been observed for different systems. ${ }^{4,5}$

\section{A BESSEL UNIFORM ASYMPTOTIC INTEGRATION}

\section{A. Introduction}

The integral given by Eq. (1.1) is the fundamental expression which must be evaluated to calculate an $S$-matrix element. Since the Bessel functions are usually defined over a $(-\pi, \pi)$ interval, we will make a change of variable and consider an integral of the form

$$
I=(2 \pi)^{-1} \int_{-\boldsymbol{r}}^{r} g(x) e^{i f(\alpha, x)} d x
$$

where the integration is over a $2 \pi$ interval rather than a unit interval. For our systems $g(x)$ is periodic in $x$ and $f(\alpha, x)$ has the property that $f(\alpha, x+2 \pi)$ equals $f(\alpha, x)-2 k \pi$ and $f^{\prime}(\alpha, x)$ vanishes at two points in the interval $-\pi<x \leq \pi$. From these properties it can easily be shown that the value of $I$ in (2.1) is unaltered when the integration domain is changed from $-\pi<x \leq \pi$ to $a<x \leq a+2 \pi$ ( $a$ is real).

The derivation of the Bessel uniform approximation is patterned after the method of Chester, Friedman, and Ursell ${ }^{9}$ for Airy uniform approximations, except we map $f(\alpha, x)$ onto a function of the form ${ }^{10}$

$$
f(\alpha, x)=-\zeta \cos y-k y+A,
$$

instead of onto a cubic polynomial in $y$. In Eq. (2.2) $k$ is intended to be an integer, $A$ and $\zeta$ are real, and $\zeta \geqslant 0$.

It should be emphasized that (2.2) is a mapping and not the first few terms of an infinite expansion of $f(\alpha, x)$. Just as in the case of the mapping onto a cubic, ${ }^{6,9}$ where it was necessary that the stationary phase points of $f(\alpha, x)$ in the interval $(-\pi<x \leq \pi)$ could be made to correspond to those of the new (cubic) function of $y$ in the interval $(-\infty<y<\infty),{ }^{9}$ it is necessary that the stationary phase points of $f(\alpha, x)$ in the interval $(-\pi<x \leq \pi)$ be made to correspond to those in the new domain of integration $(a<y \leq a+2 \pi)$. Inasmuch as the value of both $I$ in Eq. (2.1) and of the $I$ expressed as our integral over $y$ is invariant to any shift in the lower limit, as long as the upper limit is similarly shifted, we may set, without loss of generality the new limits as $-\pi$ and $\pi$ : 
$I=(2 \pi)^{-1} \int_{-\pi}^{\pi} g[x(y)](d x / d y) \exp [-i(\zeta \cos y+k y-A)] d y$

A few additional remarks on the Airy and Bessel uniform approximations may be in order. In applying the method of stationary phase one assumes that most of the contribution to the integral arises from regions near the stationary phase points of $\exp [i f(\alpha, x)]$. In the Airy uniform approximation one assumes that the range of integration may be expanded to $-\infty<y<\infty$, since the regions outside the neighborhoods of the stationary phase points do not contribute significantly. When these other regions do contribute significantly the Airy uniform approximation breaks down. The Bessel uniform approximation avoids this problem by retaining a finite interval of integration.

A mapping of this form with $k=0$ was applied to glory scattering by Berry. ${ }^{11}$

\section{B. Excitation $k \geq 0$}

Stationary phase points occur when

$$
\zeta \sin y=k
$$

which has two roots in $[-\pi, \pi]$. The mapping is one-to-one if the stationary phase points correspond.

For classically allowed transitions the correspondence would be

$$
\begin{aligned}
& x=x_{+}-y=\sin ^{-1}(k / \zeta) \equiv \theta, \quad 0 \leq \theta \leq \pi / 2, \\
& x=x_{-}-y=\pi-\theta,
\end{aligned}
$$

an equation which defines $\theta$. The fact that $x_{+}$and $x$. are real then implies that $\zeta \geq k$ for these classically allowed transitions.

From Eqs. (2.2) and (2.4) one obtains a sum and difference,

$$
\begin{aligned}
& \frac{1}{2}\left[f_{-}+f_{+}\right]=A-\frac{1}{2} k \pi, \\
& \frac{1}{2}\left[f_{-}-f_{+}\right]=\left(\zeta^{2}-k^{2}\right)^{1 / 2}-k \cos ^{-1}(k / \zeta),
\end{aligned}
$$

where $f_{-}=f\left(\alpha, x_{-}\right)$and $f_{+}=f\left(\alpha, x_{+}\right)$are real quantities. The fact that the right-hand side of (2.5) is nonnegative implies that $f_{-}$is a local maximum $\left(f_{-}^{\prime \prime}<0\right)$ and $f_{+}^{\prime}$ is a local minimum $\left(f_{+}^{\prime \prime}>0\right)$.

For classically nonallowed transitions the correspondence is

$$
\begin{aligned}
& x=x_{+}-y=\frac{1}{2} \pi+i \cosh ^{-1}(k / \zeta) \equiv \frac{1}{2} \pi+i \bar{\theta}, \quad \bar{\theta} \geq 0, \\
& x=x_{-} \rightarrow y=\frac{1}{2} \pi-i \bar{\theta},
\end{aligned}
$$

an equation which defines $\vec{\theta}$. For this classically nonallowed transition $x_{+}$and $x_{-}$are complex, a fact which implies that $0 \leq \zeta \leq k$.

The equations corresponding to Eqs. (2.5) are, for this classically nonallowed case, ${ }^{12}$

$$
\begin{aligned}
& \frac{1}{2}\left[f_{-}+f_{+}\right]=A-\frac{1}{2} k \pi, \\
& \frac{1}{2}\left[f_{-}-f_{+}\right]=-i\left(k^{2}-\zeta^{2}\right)^{1 / 2}+i k \cosh ^{-1}(k / \zeta) .
\end{aligned}
$$

Now $f_{-}$and $f_{+}$are complex with $f_{-}=f_{+}^{*}$ and causes their difference to be pure imaginary. Since the right-hand side of (2.7) has as its range the positive imaginary axis, one may deduce that $\operatorname{Im}\left(f_{-}\right)$ $>0$ and $\operatorname{Im}\left(f_{+}\right)<0$.

Expanding $(d x / d y) g$ of Eq. (2.3) as ${ }^{13}$

$$
(d x / d y)_{g}=p_{0} \cos y+q_{0} \sin y,
$$

and substituting Eqs. (2.4) into this expression, one obtains

$$
\begin{aligned}
& p_{0}=\frac{1}{2} \frac{\left[g_{+}(d x / d y)_{+}-g_{-}(d x / d y)-\right]}{\cos \theta}, \\
& q_{0}=\frac{1}{2} \frac{\left[g_{+}(d x / d y)_{+}-g_{-}(d x / d y)_{-}\right]}{\sin \theta} .
\end{aligned}
$$

The values for $(d x / d y)_{+}$and $(d x / d y)_{-}$may be obtained by differentiating Eq. (2.2) twice and inserting the stationary phase conditions. One obtains

$$
(d x / d y)_{ \pm}=\left( \pm \zeta \cos \theta / f_{ \pm}^{\prime \prime}\right)^{1 / 2} \text {. }
$$

Upon making use of readily derived expressions for the Bessel functions of integer order ${ }^{14 a}$

$$
\begin{aligned}
(k / \zeta) J_{k}(\zeta)= & \left(i^{k} / 2 \pi\right) \int_{-\pi}^{\pi} \sin (y) \\
& \times \exp [i(-\zeta \cos y-k y)] d y
\end{aligned}
$$

and

$$
J_{k}^{\prime}(\zeta)=\left(i^{k} / 2 \pi i\right) \int_{-r}^{r} \cos (y) \exp [i(-\zeta \cos y-k y)] d y,
$$

Eq. (2.3) reduces to ${ }^{15}$

$$
I=(-i)^{k} \exp (i A)\left[(k / \zeta) q_{0} J_{k}(\zeta)+i p_{0} J_{k}^{\prime}(\zeta)\right] .
$$

With the aid of Eqs. (2.9) and (2.10) the final result for the integral is obtained:

$$
\begin{aligned}
I= & \frac{1}{2} \zeta^{1 / 2} e^{i \mathrm{~A}}\left\{(\cos \theta)^{1 / 2}\left[g_{+} /\left(f_{+}^{\prime \prime}\right)^{1 / 2}+g_{-} /\left(-f_{-}^{\prime \prime}\right)^{1 / 2}\right] J_{k}(\zeta)\right. \\
& \left.+i(\cos \theta)^{-1 / 2}\left[g_{+} /\left(f_{+}^{\prime \prime}\right)^{1 / 2}-g_{-} /\left(-f_{-}^{\prime \prime}\right)^{1 / 2}\right] J_{k}^{\prime}(\zeta)\right\},
\end{aligned}
$$

where $A=\frac{1}{2}\left[f_{+}+f_{-}\right]$.

The expression for $I$ for the classically nonallowed case is of a similar derivation, and is given by

$$
\begin{aligned}
I= & \zeta^{1 / 2} \exp (i \bar{A})_{g}\left(f^{\prime \prime}\right)^{-1 / 2}\left\{\cos \gamma J_{k}(\zeta)(\sinh \bar{\theta})^{1 / 2}\right. \\
& \left.-i \sin \gamma J_{k}^{\prime}(\zeta)(\sinh \bar{\theta})^{-1 / 2}\right\}
\end{aligned}
$$

where $2 \gamma=2 \alpha-\bar{\beta}-\frac{1}{2} \pi$.

The above result took cognizance of the Schwartz reflection principle ${ }^{16}$ which allows one to write

$$
g_{t}=g e^{ \pm i \alpha} \text { and } f_{ \pm}^{\prime \prime}=f^{\prime \prime} e^{ \pm i \bar{\beta}} \text {. }
$$




\section{De-excitation $k<0$}

The procedure is identical to that of $k \geqslant 0$, except the mapping correspondence for the classically allowed transitions is given by

$$
\begin{array}{ll}
x=x_{+}, & y=-\sin ^{-1}(|k| / \zeta)=-\theta, \quad 0 \leq \theta \leq \frac{1}{2} \pi, \\
x=x_{-}, & y=-\pi+\theta
\end{array}
$$

and for the classically nonallowed transitions by

$$
\begin{aligned}
& x=x_{+}, y=-\frac{1}{2} \pi+i \cosh ^{-1}(|k| / \zeta)=-\frac{1}{2} \pi+i \bar{\theta}, \bar{\theta} \geq 0 \\
& x=x_{-}, y=-\frac{1}{2} \pi-i \vec{\theta}_{0}
\end{aligned}
$$

Making use of the fact that $J_{-k}(\zeta)=(-1)^{k} J_{k}(\zeta)$ and $J_{-k}^{\prime}(\zeta)=(-1)^{k} J_{k}^{\prime}(\zeta)$ one obtains the identical equations of Sec. II B for Eqs. (2.5), (2.7), (2.12), and (2.13) where $k$ is replaced by $|k|$.

\section{UNIFORM APPROXIMATION TO THE $S$ MATRIX}

In the semiclassical theory one usually describes the motion of a system by a radial coordinate $R$, its conjugate momentum $P$, and the action angle variables $2 \pi\left(n+\frac{1}{2}\right)$ and $w$. At an initial precollision time $t_{i}$, the interaction of the two species is negligible. Similarly, at a postcollision time $t_{f}$ the interaction of the two species (which may be different than the original species) is again negligible. The dynamical variables at $t_{i}$ and $t_{f}$ are denoted by a subscript $i$ and $f$, respectively.

The $S$-matrix element for a transition from a state $n_{i}$ to a state $m$ is given by ${ }^{12, b}(3.1)$ for one internal degree of freedom $w$

$$
S_{m n_{i}}=\int_{0}^{1}\left[d \bar{w}_{f} / d \bar{w}_{i}\right]^{-1 / 2} \exp (i \Delta) d \bar{w}_{f} .
$$

$\bar{w}_{\gamma}$ is related to the angle variable $w_{\gamma}$ by

$$
\bar{w}_{\gamma}=w_{\gamma}-\mu_{\gamma} \nu_{\gamma} R_{\gamma} / P_{\gamma}, \quad \gamma=i, f,
$$

where $\mu_{\gamma}$ is the reduced mass for the translational motion and $\nu_{\gamma}$ is the frequency of the periodic motion.

For inelastic collisions the phase $\Delta$ is given by

$$
\Delta=2 \pi\left(n_{f}-m\right) \bar{w}_{f}-\int_{t_{i}}^{t_{f}}[2 \pi w(t) \dot{n}(t)+R(t) \dot{P}(t)] d t
$$

where the dot denotes differentiation with respect to time and the integration is performed along the trajectory.

The stationary phase points of the integrand are given by $d \Delta / d w_{f}=0$ and may be found by differentiating Eq. (3.3). One obtains

$$
d \Delta / d \bar{w}_{f}=2 \pi\left[n_{f}\left(\bar{w}_{f}\right)-m\right]=0 .
$$

If one defines a new variable $\bar{q}_{f}$,

$$
\bar{q}_{f}=2 \pi \bar{w}_{f}-\pi
$$

the limits of the integral given in Eq. (3.1) are altered to $[-\pi, \pi]$ and hence the integral is of the type given in Eq. (2.1).

Differentiating Eq. (3.4) and employing Eq. (3.5) it can be seen that

$$
g_{ \pm}\left( \pm f_{ \pm}^{\prime \prime}\right)^{-1 / 2}=\left(2 \pi p_{ \pm}\right)^{1 / 2},
$$

where $p_{ \pm}$are the classical probabilities given by

$$
p_{ \pm}=\left(\left|d \bar{n}_{f} / d \bar{w}_{1}\right|\right)_{ \pm}^{-1} \text {. }
$$

Upon making these identifications for use in Eq. $(2.12)$ for the classically accessible case, one obtains for the $S$ matrix element

$$
\begin{aligned}
S_{m n_{i}}= & \frac{1}{2}(2 \pi)^{1 / 2} \zeta^{1 / 2} \exp (i A)\left\{\left(p_{+}^{1 / 2}+p_{-}^{1 / 2}\right)(\cos \theta)^{1 / 2} J_{k}(\zeta)\right. \\
& \left.+i\left(p_{+}^{1 / 2}-p_{-}^{1 / 2}\right)(\cos \theta)^{-1 / 2} J_{k}^{\prime}(\zeta)\right\}
\end{aligned}
$$

or for the transition probability $P$

$$
\begin{aligned}
P= & |S|^{2}=\frac{1}{2} \pi \zeta\left\{\left(p_{+}^{1 / 2}+p_{-}^{1 / 2}\right)^{2} J_{k}^{2}(\zeta) \cos \theta\right. \\
& \left.+\left(p_{+}^{1 / 2}-p_{-}^{1 / 2}\right)^{2} J_{k}^{\prime 2}(\zeta) / \cos \theta\right\}
\end{aligned}
$$

where

$$
\begin{aligned}
& A=\frac{1}{2}\left[\Delta_{\max }+\Delta_{\min }\right] \\
& k=|n-m|, \\
& \frac{1}{2}\left[\Delta_{\max }-\Delta_{\operatorname{mln}}\right]=\left(\zeta^{2}-k^{2}\right)^{1 / 2}-k \cos ^{-1}(k / \zeta),
\end{aligned}
$$

and

$$
\cos \theta=\left[1-(k / \zeta)^{2}\right]^{1 / 2}
$$

For the classically inaccessible case one obtains from $\mathrm{Eq}$. (2.13)

$$
\begin{aligned}
S_{m n i}= & (2 \pi)^{1 / 2} \zeta^{1 / 2} \exp (i \bar{A}) p^{1 / 2}\left\{\cos \left(\frac{1}{2} \beta-\frac{1}{4} \pi\right) J_{k}(\zeta)\right. \\
& \left.(\sinh \bar{\theta})^{1 / 2}-i \sin \left(\frac{1}{2} \beta-\frac{1}{4} \pi\right) J_{k}{ }^{\prime}(\zeta)(\sinh \bar{\theta})^{-1 / 2}\right\}
\end{aligned}
$$

and

$P=\pi p \zeta\left\{(1+\sin \beta) J_{k}^{2}(\zeta) \sinh \bar{\theta}+(1-\sin \beta) J_{k}^{\prime 2}(\zeta) / \sinh \bar{\theta}\right\}$,

where $p, \beta$, etc. are defined by

$$
\begin{aligned}
& \frac{1}{2}\left[\Delta_{-}-\Delta_{+}\right]=i \varphi, \quad \varphi>0, \\
& -\varphi=\left(k^{2}-\zeta^{2}\right)^{1 / 2}-k \cosh ^{-1}(k / \zeta), \\
& \left(d \bar{n}_{f} / d \bar{w}_{l}\right)_{ \pm}=p^{-1} e^{ \pm i \beta}, \\
& \sinh \bar{\theta}=\left[(k / \zeta)^{2}-1\right]^{1 / 2} .
\end{aligned}
$$

\section{LIMITING CASES}

\section{A. Stationary Phase Points Far Apart}

In the limit that the difference in the phases become large, Eq. (3.12) becomes

$$
\frac{1}{2}\left[\Delta_{-}-\Delta_{+}\right] \sim \zeta-\frac{1}{2} k \pi \text {. }
$$

The asymptotic expansions for large arguments of $J_{k}(\zeta)$ and $J_{k}^{\prime}(\zeta)$ are given by ${ }^{1 \ell b}$

$$
\begin{aligned}
& J_{k}(\zeta) \sim(2 / \pi \zeta)^{1 / 2} \cos \left(\zeta-\frac{1}{2} k \pi-\frac{1}{4} \pi\right), \\
& J_{k}^{\prime}(\zeta) \sim-(2 / \pi \zeta)^{1 / 2} \sin \left(\zeta-\frac{1}{2} k \pi-\frac{1}{4} \pi\right) .
\end{aligned}
$$


TABLE I. Ratio of $[k / 2]^{1 / 3} J_{k}(k)$ to $\mathrm{Ai}(0)$ for various $k{ }^{2}$

\begin{tabular}{lc}
\hline \hline$k$ & Ratio \\
\hline 1 & 0.9838 \\
2 & 0.9938 \\
3 & 0.9965 \\
4 & 0.9977 \\
6 & 0.9987 \\
8 & 0.9991 \\
\hline \hline
\end{tabular}

${ }^{\mathrm{a}} \mathrm{Ai}(0)=0.355028$.

When they are introduced into $\mathrm{Eq} .(3.9)$, one obtains

$$
P \sim p_{+}+p_{-}+2\left(p_{+} p_{-}\right)^{1 / 2} \cos \left(2 \zeta-k \pi-\frac{1}{2} \pi\right),
$$

Upon noting that the $\cos \theta$ in (3.13) approaches unity for large $\zeta$.

Application of Eq. (4.1) then yields the usual semiclassical result

$$
P \simeq p_{+}+p_{-}+2\left(p_{+} p_{-}\right)^{1 / 2} \sin \left(\Delta_{-}-\Delta_{+}\right)
$$

\section{B. Stationary Phase Points Close Together}

When the stationary phase points are close together one may approximate $n_{f}\left(n_{i}, \bar{w}_{i}\right)$ as a quadratic about a particular $\bar{w}_{i}=\bar{w}_{i}^{e}$ at which $n_{f}$ is an extremum, i.e.,

$$
n_{f}\left(n_{i}, \bar{w}_{i}\right) \cong a\left(\bar{w}_{i}-\bar{w}_{i}^{e}\right)^{2}+n_{f}^{e} \text {. }
$$

In some interval about $\bar{w}_{i}^{e}$ the value of $d \bar{w}_{f} /$ $d \bar{w}_{i}\left(\equiv \bar{w}^{\prime}\right)$ may be considered a constant. This approximation enables one to calculate $\Delta$ by

$$
\Delta\left(n_{i}, \bar{w}_{i}\right)=\int 2 \pi\left[n_{f}\left(n_{i}, \bar{w}_{i}\right)-m\right] \bar{w}^{\prime} d \bar{w}_{i} .
$$

The stationary phase points are found by solving $n_{f}\left(n_{i}, \bar{w}_{i}\right)=m$. The phase $\Delta$ and classical probability $p$ are given by Eqs. (4.5) and (3.7), respectively.

In the limit that the stationary phase points coalesce, $\zeta$ approaches $k$ and one obtains for the probability

$$
P=\left[\frac{1}{2} k\right]^{2 / 3}\left[(2 \pi)^{4} \bar{w}^{\prime} / a^{2}\right]^{1 / 3} J_{k}^{2}(k)
$$

in this coalescence limit.

The corresponding expression for the probability using the Airy uniform approximation is given by

$$
P=\left[(2 \pi)^{4} \bar{w}^{\prime} / a^{2}\right]^{1 / 3} \mathrm{Ai}^{2}(0) .
$$

Comparing Eqs. (4.6) and (4.7), one sees they are equivalent if

$$
\mathrm{Ai}(0) \simeq[k / 2]^{1 / 3} J_{k}(k)
$$

A comparison of the two sides of Eq. (4.8) are given in Table I and clearly shows that this relation holds.

\section{Near Elastic Collision}

Using the same type of approximation as given in Eq. (1.2), one obtains for the Bessel uniform probability

$$
P \simeq J_{0}^{2}(\epsilon),
$$

which in the limit of $\epsilon$ approaching zero goes to unity (the correct result) since $J_{0}(0)=1$. This behavior is in contrast to the Airy uniform expression (1.3) which became infinite when $\epsilon \rightarrow 0$.

\section{Highly Classically Nonallowed Transitions}

The classically nonallowed transitions have $0 \leq \zeta \leq k$ with $\zeta$ approaching zero as the transitions become less probable. Therefore, Eq. (3.16) may be approximated by

$$
-\varphi \simeq k-k \ln (2 k / \zeta)
$$

in this region of highly classically nonallowed transitions. For small $\zeta, J_{k}(\zeta)$, and $J_{k}^{\prime}(\zeta)$ may be approximated by

$$
\begin{aligned}
& J_{k}(\zeta) \simeq\left(\frac{1}{2} \zeta\right)^{k} / k ! \\
& J_{k}^{\prime}(\zeta) \simeq\left(\frac{1}{2} \zeta\right)^{k-1} / 2(k-1) ! .
\end{aligned}
$$

Substituting Eqs. (4.11) into the expression for the probability given in Eq. (3.15) one obtains

$$
P \simeq 2 k \pi p\left(\frac{1}{2} \zeta\right)^{2 k} /(k !)^{2} .
$$

From Eq. (4.10) one finally obtains

$$
P \simeq \eta p e^{-2 \varphi}
$$

where

$$
\eta=2 k \pi(k / e)^{2 k} /(k !)^{2} .
$$

This expression differs from the usual semiclassical expression for nonallowed transitions by the factor $\eta$. This factor does, however, approach unity as $k$ becomes large. Indeed, even for $k=1$, $\eta=0.8503$ and so does not introduce a major error into the result. Typically, the error produced is less than that introduced by $\eta$, but will cause the probabilities to be less than the exact quantum.

\section{APPLICATION TO A NONREACTIVE SYSTEM}

We shall apply the uniform approximation derived in Sec. III to the collinear, nonreactive, atom-diatom system given by Secrest and Johnson. ${ }^{17}$ Their exact quantum mechanical results for the transition probabilities will be compared to those calculated with the Airy and Bessel uniform approximation. The Hamiltonian is the same as given by Eq. (1) of Ref. 1(e), that is

$$
\begin{aligned}
H= & (2 \mu)^{-1} P^{2}+\left(n+\frac{1}{2}\right) \\
& +\exp \left\{-\alpha\left[R-(2 n+1)^{1 / 2} \sin 2 \pi w\right]\right\}=E,
\end{aligned}
$$

where $R$ is the distance from the incoming atom to 
TABLE II. Comparison of exact quantum with Airy and Bessel uniform approximations.

\begin{tabular}{|c|c|c|c|c|c|c|c|}
\hline$\alpha$ & $\begin{array}{c}\text { System }^{2} \\
\mu\end{array}$ & $E$ & $\begin{array}{l}\text { Transition } \\
\qquad n \rightarrow m\end{array}$ & Type & $\begin{array}{l}\text { Airy } \\
\text { uniform }^{b, c}\end{array}$ & $\begin{array}{c}\text { Bessel } \\
\text { uniform }^{b, d}\end{array}$ & $\begin{array}{c}\text { Exact } \\
\text { quantum }^{\text {b,e }}\end{array}$ \\
\hline 0.114 & $1 / 5$ & 3.0 & $0-1$ & Nonallowed & $7.59(-4)$ & $6.76(-4)$ & $7.06(-4)$ \\
\hline 0.114 & $1 / 2$ & 3.0 & $0-2$ & Nonallowed & $9.14(-10)$ & $8.54(-10)$ & $9.03(-10)$ \\
\hline 0.114 & $1 / 2$ & 3.8 & $0-1$ & Nonallowed & $4.69(-5)$ & $4.12(-5)$ & $4.30(-5)$ \\
\hline 0,114 & $1 / 2$ & 3.8 & $1-1$ & Allowed & $4.707^{\mathrm{P}}$ & 1.000 & 1.000 \\
\hline 0.300 & $2 / 3$ & 3.0 & $1-1$ & Allowed & $1.457^{\mathrm{f}}$ & 0.966 & 0.977 \\
\hline 0.300 & $2 / 3$ & 4.0 & $0-1$ & Nonallowed & $1.08(-1)$ & $1.03(-1)$ & $1.08(-1)$ \\
\hline 0.300 & $2 / 3$ & 6.0 & $1-1$ & Allowed & $2.23(-1)$ & $2.33(-1)$ & $2.24(-1)$ \\
\hline 0.300 & $2 / 3$ & 6.0 & $1-2$ & Allowed & $3.49(-1)$ & $3.44(-1)$ & $3.45(-1)$ \\
\hline 0.300 & $2 / 3$ & 10.0 & $0-1$ & Allowed & $2.11(-1)$ & $2.05(-1)$ & $2.18(-1)$ \\
\hline
\end{tabular}

${ }^{a} \alpha, \mu$ and $E$ are the dimensionless parameters in the model of Secrest and Johnson (our $E$ is their $E / 2$ ).

Number in parentheses is power of 10.

${ }^{c}$ Calculated from Eqs. (4.14) or (4.16) of Connor and Marcus. ${ }^{\text {tc }}$

${ }^{\mathrm{d}}$ Calculated from Eqs. (3.9) or (3.15).

${ }^{8}$ Exact quantum mechanical results of Secrest and Johnson. ${ }^{17}$

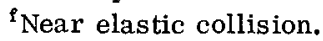

the center of mass of the harmonic oscillator and $P$ is the conjugate momentum; $\alpha, \mu, E$ are parameters given in Table II; $2 \pi\left(n+\frac{1}{2}\right)$ and $w$ are the action and angle variables for the harmonic oscillator.

Hamilton's equations of motion were numerically integrated with a large initial $R$ and the oscillator initially in the $n_{i}$ state. The initial radial momentum $P_{i}$ is then given by $-\left[2 \mu\left(E-n_{i}-\frac{1}{2}\right)\right]^{1 / 2} \cdot \bar{w}_{i}$ was then varied until the stationary phase condition $n\left(\bar{w}_{i}, n_{i}\right)=m$ was satisfied. There were two such points for the above system. The phase $\Delta$ was obtained by integrating

$$
\dot{\Delta}=2 \pi \dot{m}+R \dot{P}
$$

along with Hamilton's equations. The classical probability $p$ as given by Eq. (3.7) was evaluated at the two stationay phase points.

The Bessel uniform probabilities were then calculated using Eq. (3.9) for the classically accessible transitions and Eq. (3.15) for the classically inaccessible transitions. Equations $(4.14)$ and (4.16) of Ref. 1(c) were used to calculate the Airy uniform probabilities. The results are given in Table II.

\section{DISCUSSION}

It has been shown in Sec. IV that the Airy uniform approximation and Bessel uniform approximation are essentially equivalent except for the nearelastic collision. This fact also agrees with the results given in Table II, where for near elastic collisions the Airy formula gives gross deviations from the exact quantum probabilities but the Bessel formula gives good results.

Whereas in the past it was necessary to calculate the $S$ matrix integral directly for large probabilities, ${ }^{1 \mathrm{~d}}$ it is hoped that now one equation will serve to calculate probabilities of all magnitudes. The extension of this method to several dimensions is currently being explored. ${ }^{18}$

*Supported in part by a grant from the National Science Foundation.

'(a) R. A. Marcus, Chem. Phys. Lett. 7, 525 (1970); (b) R. A. Marcus, J. Chem. Phys. 54, 3965 (1971); (c) J. N. L. Connor and R. A. Marcus, J. Chem. Phys. 55, 5636 (1971); (d) W. H. Wong and R. A. Marcus, J. Chem. Phys. 55, 5663 (1971); (e) J. Stine and R. A. Marcus, Chem. Phys. Lett. 15, 536 (1972); (f) R. A. Marcus, J. Chem. Phys. 57, 4903 (1972); (g) R. A. Marcus, J. Chem. Phys. 56, 311 (1972); (h) R. A. Marcus, J. Chem. Phys. 56, 3548 (1972); (i) J. N. L. Connor, Mol. Phys. 25, 181 (1973); J. N. L. Connor, Discuss. Faraday Soc. (to be published).

${ }^{2}$ (a) W. H. Miller, J. Chem. Phys. 53, 1949 (1970); (b) J. Chem. Phys. 53, 3578 (1970); (c) J. Chem. Phys. 54, 5386 (1971); (d) Chem. Phys. Lett. 7, 431 (1970); (e) W. H. Miller and T. F. George, J. Chem. Phys. 56, 5668 (1972); (f) W. H. Miller and T. F. George, J. Chem. Phys. 57, 2458 (1972); (g) C. C. Rankin and W. H. Miller, J. Chem. Phys. 55, 3150 (1971); (h) J. D. Doll and W. H. Miller, J. Chem. Phys. 57, 5019 (1972); (i) J. D. Doll, T. F. George, and W. H. Miller, J. Chem. Phys. 58, 1343 (1973); (j) W. H. Miller and A. W. Raczkowski, Discuss. Faraday Soc. (to be published).

${ }^{3}$ Other examples of recent semiclassical work include (a) R. D. Levine and B. R. Johnson, Chem. Phys. Lett. 7, 404 (1970); (b) P. Pechukas, Phys. Rev. 181, 166 (1969); Phys. Rev. 181, 174 (1969); (c) I. L. Beigman, L. A. Varnshtein, and I. I. Sobel'man, Zh. Eksp. Teor. Fiz. 57, 1703 (1969) [Sov. Phys.-JETP 30, 920 (1970)]; (d) I. C. Percival and D. Richards, J. Phys. B 3, 315 (1970); J. Phys. B 3, 1035 (1970).

${ }^{4}$ J. M. Bowman and Aron Kuppermann, Chem. Phys. Lett. 19, 166 (1973)

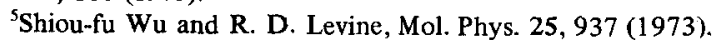

${ }^{6}$ The term "Airy uniform" used throughout this paper will refer to that uniform approximation derived in Ref. 1(c).

${ }^{7}$ R. D. Levine, Mol. Phys. 22, 497 (1971).

${ }^{8}$ The functions $f(\alpha, x)$ and $d^{2} f(\alpha, x) / d x^{2}$ may be obtained by integrating and differentiating Eq. (1.2), respectively. The points 
of stationary phase are obtained by equating Eq. (1.2) to zero and solving for $x$. These results are then substituted into the Airy uniform expression given by Eq. (3.20) of Ref. 1(c).

${ }^{9} \mathrm{C}$. Chester, B. Friedman, and F. Ursell, Proc. Camb. Philos. Soc. 53, 599 (1957).

${ }^{10}$ Approximations to the phase which are of this form have been made elsewhere in the literature, Refs. 2(b), 3(a), and 7. However, we employ instead a mapping.

${ }^{11}$ M. V. Berry, J. Phys. B 2, 381 (1969).

${ }^{12}$ Equation (2.5) may be transformed for computational convenience, to $\left[f_{-}-f_{+}\right] / 2 k=\tan \theta^{\prime}-\theta^{\prime}$, where $\theta^{\prime}=(1 / 2) \pi-\theta$, by use of Eq. (2.4). Likewise, Eq. (2.7) becomes $i\left[f_{-}-f_{+}\right] / 2 k=\tanh \bar{\theta}-\bar{\theta}$.

${ }^{13}$ This approximation differs from that of Chester, Friedman, and Ursell. Since the physical variables encountered in the semiclassical formalism are periodic, a periodic expansion would be more appropriate than a polynomial expansion. At this point Berry expanded $g(d x / d y)$ as $p_{0}+q_{0} \cos y$ which gives exactly the same results as given here.

${ }^{14}$ (a) M. Abramowitz and I. A. Stegun (Eds.), Natl. Bur. Std. (U.S.), Appl. Math. Ser. 55, 360 (1964). Equation (9.1.21), with $\theta=y+\pi$, and Eq. (9.1.27) were used. (b) Natl. Bur. Std. (US), Appl. Math. Ser. 55, 364 (1964).

${ }^{15}$ One has to make use of the following easily derivable relationships: $J_{k}(-\zeta)=(-1)^{k} J_{k}(\zeta)$ and $J^{\prime}{ }_{k}$ $(-\zeta)=-(-1)^{k} J_{k}^{\prime}(\zeta)$

${ }^{16}$ P. M. Morse and H. Feshbach, Methods of Theoretical Physics (McGraw-Hill, New York, 1953), p. 393.

${ }^{17}$ D. Secrest and B. R. Johnson, J. Chem. Phys. 45, 4556 (1966). ${ }^{18}$ H. Kreek and R. A. Marcus (unpublished). 